\title{
Alianza Estratégica Institucional entre el SIGTUR-ZULIA y la Cuenta Satélite de Turismo de Venezuela
}

\author{
Nelly Caldera de Ugarte \\ Janet Cestary ${ }^{2}$ \\ Leriz Camacaro ${ }^{3}$ \\ Ricardo Cuberos ${ }^{4}$
}

RESUMEN: Dada la importanciacreciente del turismo en el mundo y específicamente para Venezuela y en particular el estado Zulia como parte del contexto del Caribe turístico, resulta de particular interés vincular una investigación en proceso denominada SIGTUR-ZULIA con el proyecto internacional Cuenta Satélite de Turismo (CST), de allí la intención manifiesta de plantear en el presente artículo los aspectos relevantes paradar viabilidad a una "Alianza Estratégica Interinstitucional" fundamentada a través del conocimiento y experiencias generadas en esta investigación, para establecer una cooperación recíproca en la elaboración de la CST de Venezuela, con la incorporación del SIGTUR-ZULIA en el referido ámbito internacional y científico del turismo.

PALABRAS CLAVES: planificación territorial, planificación económica; sistemas de información geográfica, cuenta satélite turismo, alianza estratégica interinstitucional.

1. Arquitecta. Magister Scientiarum en Planificación Integral para el Desarrollo del Turismo. Profesora-investigadora Responsabledel Programa SIGTUR-ZULIA enel Instituto de Investigaciones de la Facultad de Arquitectura y Diseño de la Universidad del Zulia, Maracaibo, Venezuela.

E-mail: ncaldera@luz.ve

2. Arquitecta.Magister Scientiarumen Planificación Integral parael Desarrol lodel Turismo. Especialistaen Arquitectura. Profesora-Coinvestigadora del Programa SIGTUR-ZULIA en el Instituto de Investigaciones de la Facultad de Arquitectura y Diseño de la Universidad del Zulia, Maracaibo, Venezuela.

E-mail: jcestary@hotmail.com

3. Arquitecta. Magister Scientiarumen Planificación Integral parael Desarrollodel Turismo. Master en Geoinformacion para la Planificacion Urbana. Profesora-Coinvestigadoradel Programa SIGTUR-ZULIA en el Institutode Investigaciones de la Facultad de Arquitectura y Diseiio de la Universidad del Zulia, Maracaibo, Venezuela.

E-mail: leriz@luz.ve

4. Arquitecto. Magister en Sistemas de Información - Director del Instituto de Investigaciones de la Facultad de Arquitectura y Diseino de la Universidad del Zulia Profesor Investigador del Programa SIGTUR-ZLLIA en ci Instituto de Investigaciones de la Facultad de Arquitectura y Diseino de la Universidad del Zulia, Maracaibo, Venezuela. E-mail: rcuberos@luz..ve 
ASSTRACT: Due to the rising concern of tourism in the world, and specifically for Venezuela and particularly to Zulia state, as part of the tourist Caribbean context, it is necessary to enail a research in process known as SIGTURZULIA along with the international project Tourism Satellite Account (TSA). The intention of the present article is to carry out the most important aspects to make a "Interinstitutional Strategic Alliance" to be feasible, based upon the knowledge and experiences generated in this research, in order to establish a reciprocal cooperation in terms of elaborating the TSA

incopporation of the SIGTUR.ZULIA in the scientific and international tourist field.

KEYWORDS: ierritorial planning, economic planning, geographical information systems, tourism satellite account, interinstitutional strategic alliance.

\section{Introducción}

El tema escogido para la elaboración del presente articulo presenta las características Institucionales relevantes del Programa de Investigación denominado "Sistema de Información Geográfica Turística para la Planificación del Turismo del Estado Zulia: SIGTUR-ZULIA 1997-2001"; que respalda la intención de cooperar, desde la academia, en el desarrollo de la Cuenta Satélite de Turismo de Venezuela (CSI). El Programa de Investigación mencionado se realiza bajo la responsabilidad académica y administrativa de un grupo de profesores de la Facultad de Arquitectura y Diseño de la Universidad del Zulia, Venezuela, dedicada en forma paralela y complementaria al estudio de los Sistemas de Información Geográfica aplicados a la Planificación Integral del Turismo, como una herramienta novedosa y eficaz para optimizar los recursos, tiempo y mejorar la calidad de la toma de decisiones por parte de sus usuarios.

Este articulo tiene como objetivo dar a conocer la intención que tiene el equipo de investigadores del SIGTUR-ZULIA de la Universidad delZulia, Venezuela de establecer una Alianza Estratégica Interinstitucional, como vía especifica para participar en la elaboración de la CST de Venezuela, conjuntamente con los entes rectores de la administración publica del Turismo, los productores de estadísticas y las fuentes de información, tanto del sector publico como privado, y en todos sus niveles nacional, regional y local, con competencia e interés en el desarrollo de la actividad turística. A continuación se presentan los objetivos tecnológicos, académicos e institucionales del programa de investigación; la Plataforma Institucional que lo respalda y losaspectos conceptuales de la propucsta Alianza Estrateigica Institucional

\section{Programa de Investigación "Sistema de Información Geográfica Turística para la Planificación del Turismo del Estado Zulia": SIGTUR-ZULIA 1997-2001}

Los objetivos siguientes constituyen la motivación básicapara el acercamicnto Institucional de LUZ con las Cuentas Satélite de Turismo que en la actualidad en este país, se encuentran en periodo de gestación por parte de la (ANT) Corporación Venezolana de Turismo (CORPOTURISMO)

\section{Objetivos Tecnológicos}

of Venezuela with the

Crear una Base de Información Turística Automatizada y Georeferenciada, organizada por municipios, de los recursos naturales y culturales relevantes y diferenciados del estado Zulia, para delimitar y calificar funcionalmente las áreas de interés turístico estatal, para ser utilizada como una herramienta novedosa y eficaz de la informática en nuestro país, a fin de mejorar la calidad de la toma de decisiones en el proceso de planificación territorial del turismo, por parte de los diferentes usuarios SIGTUR-ZULIA (2000).

\section{Objetivos Académicos}

a) desarrollar una plataforma deinvestigación especifica parael Programa Académico de Maestría en "Planificación Integral para el Desarrollo del Turismo", que se ofrece en la División de Estudios para Graduados de la Facultad de Arquitectura y Diseño de LUZ, creada desde el año 1997, para el desarrollo del Trabajo de Grado y del curso denominado "Sistemas de Información Geográfica aplicados al Turismo";

b) apoyar la Investigación en el rírea de los Sistemas de Información Geográfica (SIG), delPrograma Académicus de Especialización y de Maestría en "Informática en Arquitectura", que se ofrece en la División de Estudios para Graduados de la Facultad de Arquitectura y Diseño de LUZ, para el desarrollo del Trabajo de Grado y de otros cursos de su curriculo.

\section{Objetivos Institucionales}

a) vincular las actividades de investigación que realizan los docentes/investigadores de las secciones de "Patrimonio y Turismo" y de "Sistemas de Información", del Instituto de investigaciones, y las de docentes/investigadores adscritos a los programas de postgrado antes mencionados; 
b) intercambiar experiencias académicas con otrasuniversidades, bajo la modalidad de Tutorías de Trabajos de Grado, con temáticas relacionadas al SIGTURZULIA;

c) "contribuir con la Investigación, a lasolución de problemasregionales, nacionales y al mejor conocimiento de la realidad venezolana" (Universidad del Zulia 1996), cn este caso en el campo de la planificación del turismo, mediante la suscripción de Convenios para Alianzas Estratégicas, con las Instituciones encargadas dela administración publica del Turismo y/ocon otros entes interesados en el avance del conocimiento científico del Turismo, para contribuir desde la Academia con cl desarrollo social y económico de esta actividad, que se encuentra en fase incipiente en nuestra localidad (Investigación Aplicada).

\section{La Plataforma Institucional del Programa de Investigación SIGTUR-ZULIA}

El proceso de descentralización administrativa y transferencias de competencias en materia turística, desde cl gobierno central hacia las Alcaldías y las Gobernaciones de Estado, llevado a cabo en Venezucla desde 1996, constituyen el mejor estímulo para inducir desde la academia la participación de los entes oficiales competentes en el desarrollo del Turismo en el desarrollo de la primera fase de esta investigación aplicada al Municipio Maracaibo.

La primera fase del programa de investigación, fue dedicada a la definición del Modelo Teórico Metodológico Turístico de la Planificación Territorial de Turismo (MOTEPLANTUR), base conceptual para la construcción del Modelo del SIGTUR-MUNICIPAL, Prototipo del "SIGTUR-ZULIA", utilizando como estrategia el intercambio de conocimientos y necesidades entre el grupo de investigación y algunos representantes de entes públicos del turismo, interesados en el sistema.

A través del intercambio, producto de estas relaciones institucionales, se pudo confrontar la idoneidad de la aplicación de las tecnologías de los Sistemas de Información Geográfica para almacenar, actualizar y manipular en forma automatizada, la cuantiosa y diversificada data turística, de utilidad a la academia y a las instituciones consultadas, dada que al ser concebido el SIGTUR-ZULIA como un "sistema abierto", adquiere la propicdad técnica de agregar y/o sustraer data o Registros de Información Turística, para dar respuestas descriptivas y predictivas, acordes a las necesidades particulares de los usuarios Institucionales.

Se inicia la construcción del Sistema SIGTUR-MARACAIBO, basado en el MOTEPLANTUR que define y sistematiza las clasificaciones y definiciones operacionales de catorce subsistemas de variables establecidos, que orientan la organización normalizada y definen el lenguaje técnico para la recolección de los datos correspondientes a los Registros Turísticos requeridos por los usuarios de la academia y de los entes públicos, a su vez son lo suficiente consistentes para ser comparables en el tiempo y en el espacio, por otros agentes administradores de turismo en el ámbito municipal, regional y nacional de nuestro país.

Este aspecto de "conceptualización" de esta investigación, presenta gran afinidad con cl Proyecto Internacional CST, cs por ello que vemos con pertinenci la posibilidad de establecer una ALIANZA ESTRATÉGICA, cuyo intercambio de conocimientos con un objetivo comum,permita incorporar al sistema en construcción, por asociación, otros atributos de "orden económico según la CST", que permitan contabilizar a través de algunos indicadores monetarios y no monetarios, la dimensión económica de la actividad turística y evaluar su repercusión en el espacio turístico municipal que se estudia. Por ejemplo, medir la inversión en servicios turísticos hoteleros, de transporte entre otros, o como lo refiere Miller (2000), el crecimiento del cmpleo en la industria turística, y del PIB turístico, que también pueden estar asociadas estas mediciones a su localización concentrada o dispersaen el territorio.

Desde el año 1998 elPrograma de Investigación, cuentacon unaPlataforma Institucional, en la cual se tiene definida las distintas modalidades de participación.

Un equipo técnico permanente y multidisciplinario de profesionales universitarios especialistas en los campos de la Informática (SIG) y la Planificación Integral del Turismo, adscritos a tres dependencias internas de la Facultad de Arquitectura y Diseño de LUZ:

a) División de Estudios para Graduados;

b) Instituto de Investigaciones;

c) Escuela de Arquitectura.

Apoyo económico de otras dependencias de LUZ:

a) Coordinación Central de Estudios para Graduados;

b) Consejo de Desarrollo Científico y Humanístico.

Colaboradores, de organismos externos a la Universidad del Zulia, basados en Convenios y Acuerdos Interinstitucionales, algunos efectivos y otros en proceso de consolidación:

a) Alcaldía del Municipio Maracaibo (Servicios de Información);

b) Corporación Zuliana de Turismo (Personal profesional);

c) Ministerio de Infraestructuras Región Zuliana (Servicios de Información);

d) Oficina Central deEstadísticas e InformáticadelZulia,OCEI-ZULIA(Generación y mantenimiento de Registros Turísticos en SIG);

e) Corporación Venezolana de Turismo, CORPOTURISMO (Servicios de Información y de cooperación para realizar otras aplicaciones específicas del SIGTUR, acorde a sus necesidades)

La experiencia acumulada que se deriva de las relaciones institucionales referidas, respalda al equipo de investigadores del SIGTUR-ZULIA, para presentar una propuesta Institucional de ALIANZA ESTRATÉGICA, que ha de vincular a 
la academia interesada en el desarrollo sustentable de la actividad turística en Venezuela, con este proyecto internacional CST y en particular integrar al contexto Turístico Internacional, el desarrollo del Turismo en el Estado -Región Zuliana y así contribuir a proporcionar a la Industria información precisa, relevante y oportuna.

\section{La Propuesta "Una Alianza Estratégica Institucional entre la Academia y la Administración Publica del Turismo para el desarrollo de la CST de Venezuela: caso de referencia Estado - Región Zuliana"}

Los objetivos, beneficios y la baseconceptual de la propuesta para establecer una Alianza Estratégica son los siguientes.

\section{Objetivos Tecnológicos}

Integrar a la Base de Información Turística Automatizada y Georeferenciada (bitag) del SIGTUR-ZULIA, algunos Registros Estadísticos Económicos, en los términos que establece la CST para Venezuela.

\section{Objetivos Institucionales}

Establecer una Alianza Estratégica Interinstitucional, a través del poder del "conocimiento", dentro del área de las respectivas competencias y actividades, para la cooperación recíproca en la elaboración de la Cuenta Satélite de Venezuela, en consonancia a las particularidades del Programa de Investigación SIGTUR-ZULIA, garantizando el máximo aprovechamiento de los recursos humanos, tecnológicos y económicos disponibles en las Instituciones y ONG involucradas.

\section{Beneficios}

a) cooperación mutua entre las entidades encargadas de las estadísticas asociadas a la CST;

b) vinculación de la investigación universitaria a la investigación y gestión de os entes administradores del Turismo;

c) los empresarios turísticos, contarán con una herramierita de la informática que centraliza, con un lenguaje técnico común la información turística, precisa, relevante y oportuna, para mejorar la calidad de la toma de decisiones en el mercado laboral y de inversiones;

d) aprovechamiento de las fortalezas institucionales;

e) Optimización en el uso de los recursos disponibles (integración y globalización)

f) actualización de "conocimientos y experiencias": definición de campos de información uniformes y comparables en el Contexto Turístico Internacional.

\section{Base Conceptual}

En la figura 1 sepresenta el diagramaque representaen forma gráfica. La Base Conceptual de la propuesta, que se presenta en este articulo, para establecer una ALIANZA ESTRATEGICA, entrelas Instituciones, que se estimantienen pertinencia e interés para conformar la Plataforma Institucional para la elaboración de la CST de Venezuela (ver figura 1).

\section{Los Protagonistas, Nivel de Participación y Tareas Propuestas}

En la tabla 1, se presenta la propuesta que contempla a las ocho instituciones, con la delimitación del nivel de participación y las tareas a cumplir por cada una de ellas (finalidades y/o necesidades), para participar en la ALIANZA ESTRATÉGICA, conducente a lograr los objetivos y beneficios antes enunciados (OMT, 2000).

\section{Productos}

Los productos esperados de la Alianza Estratégica son:

a) algunos aspectos de la Cuenta Satélite de Venezuela: como un sistema de contabilidad turístico, que aporta la información fiable para los entes turísticos del sector público y el empresariado turístico, a ser utilizado como instrumento para la toma de decisiones, en relación con las inversiones y/o negocios turísticos, en el ámbito territorial nacional, estatal, así mismo al ofrecer un lenguaje común, es comparable en el contextointernacional de las CST de otros países del mundo;

b) la Base de Información Turística, Automatizada y Georeferenciada del Estado Zulia: con el "valor agregado científico" que adquiere al asociar la base conceptual y metodológica de las Cuentas Satélite de Turismo, al Programa de investigación SIGTUR-ZULIA, por lo tanto pasa a ser una herramienta de la informática con el valor agregado por integraralgunosindicadores económicos, para mejorar la calidad de la toma de decisiones en el proceso de planificación económica y territorial de la actividad turística, en el ámbitoestatal y además por compatibilizar con el lenguaje común de la CST, es comparable con el respectivo contexto turístico nacional e internacional. 


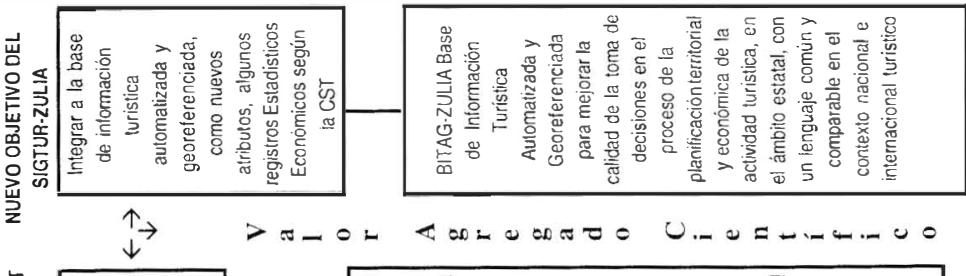

TABLA 1 - LOS PROTAGONISTAS, EL NIVEL DE PARTICIPACIÓN Y TAREAS




\begin{tabular}{|c|c|c|}
\hline Protagonistas & $\begin{array}{l}\text { Nivel de } \\
\text { Participación }\end{array}$ & Tareas (finalidades $y / 0$ necesidades) \\
\hline $\begin{array}{l}\text { Corporación Zuliana } \\
\text { Turismo /CORZUTUR }\end{array}$ & $\begin{array}{l}\text { Nacional y } \\
\text { Estata:- } \\
\text { Regional }\end{array}$ & $\begin{array}{l}\text { Eaborar Estadisticas de la Oferta y Demanda Turisticas del } \\
\text { Estado Zuía, según b establece la Ley Orgánica de Turismo } \\
\text { vigente, y ahora acorde a los parámetros de la CST de } \\
\text { Venezuela. }\end{array}$ \\
\hline $\begin{array}{l}\text { Oficina de Turismo del } \\
\text { Municipio /Maracaibo }\end{array}$ & Muricipal & $\begin{array}{l}\text { Cooperar con CORZUTUR, en a elaboración de los } \\
\text { Registros Turisticos Municipales, } \\
\text { Ordenanza respectiva sobre Tuismo vigente y ahora acorde } \\
\text { a los parämetros de la CST de Vene zuela. }\end{array}$ \\
\hline $\begin{array}{l}\text { Universidad del Zuía } \\
\text { Prograrna de hvestigación } \\
\text { SIGTUR-ZUl.A }\end{array}$ & $\begin{array}{l}\text { Internacional, } \\
\text { Nacional, Estatal } \\
\text { y Municipal }\end{array}$ & $\begin{array}{l}\text { 1. Cooperar en a elaboración de la CST para Vene zuela, } \\
\text { mediante la integración de algunos registros estadisticos } \\
\text { turisticos a a bitag del SIGTUR-MARACAIBO-ZULIA y } \\
\text { acorde a bs parámetros de la CST de Venezuela. } \\
\text { 2. Participar con carácter permanente en las Conterencias } \\
\text { CST. } \\
\text { 3. Contribuir con a formación y consolídación de un equipo } \\
\text { técnico o recuisos humanos especialistas en CST. }\end{array}$ \\
\hline OCEl- Nacional y Regional & Estatal- Regional & $\begin{array}{l}\text { Producir y surrinistrar las estadisticas básicas turisticas } \\
\text { acorde a bs parámetros de la CST de Venezuela yen } \\
\text { cooperación con CORPOTURISMO y CORZUTUR. }\end{array}$ \\
\hline $\begin{array}{l}\text { BANCO CENTRAL DE } \\
\text { Vene zuela - Naciorial } \\
\text { y Sede Regional }\end{array}$ & Estatal. Regional & $\begin{array}{l}\text { 1. Vincular los sistemas de Estad śticas Nacionales y } \\
\text { Regionales a as Estad isticas Turísticas Nacionales y locales. } \\
\text { 2. P Ioducir y yuministrar las Estadisticas Bäsicas fur'sticas } \\
\text { del Estado Zulia, acorde a bs parámetros de la CST. }\end{array}$ \\
\hline $\begin{array}{l}\text { Asociaciones de } \\
\text { Empresas Turisticas- } \\
\text { Cap tulo Regional }\end{array}$ & Estatal- Regional & $\begin{array}{l}\text { Suministrar a CORZUTUR, los datos requeridos para a } \\
\text { elaboración de bs Registros Turísticos de la Oferta y a } \\
\text { Demanda, acorde a los parámetros de la CST. }\end{array}$ \\
\hline $\begin{array}{l}\text { Organización Mundial del } \\
\text { Turismoy } \\
\text { CORPOTURISMO }\end{array}$ & $\begin{array}{l}\text { Internacional- } \\
\text { Nacional }\end{array}$ & $\begin{array}{l}\text { 1. Asistir técnicamente al equipo humano que se contorma a } \\
\text { través de esta Alianza Estratégica. } \\
\text { 2. Transterir al equipo humano bs lineamientos generales de } \\
\text { las CST, para de sarrollar bs Indicadores Estadisticos } \\
\text { Económicos, comparables intemacionamente., }\end{array}$ \\
\hline
\end{tabular}

Fuente: Elaboración Propia. SIGTUR ZULIA. Fac. Arquitectura y Diseño de LUZ (2001).

\section{Viabilidad: Recursos Humanos, Físicos y Económicos}

Es necesario resaltar la utilidad que tiene para dar viabilidad a la conformación del equipo técnico de investigadores, funcionarios, empresarios, de Venezuela y del Zulia en particular, el conocer la forma en que debe elaborarse, implementarse y mantener actualizada una CST, con la finalidad de incorporar y actualizar, según los alcances de cada institución, en la CST de nuestro país y en particular en el programa SIGTUR-ZULIA, los aspectos de la dimensión económica del Turismo que en ella se describen. También es de igual interés la oportunidad que se ofrece para tener un mejor conocimiento del significado, en este caso, de la Oferta y la Demanda Turística, 
en coherencia con la normativa de la Organización Mundial del Turismo (OMT).

El equipo técnico que se recomienda participar, está representado en los siguientes recursos humanos:

a) profesores y estudiantes del Postgrado en Planificación Integral para el Desarrollo del Turismo, de LUZ, como agente académico, que por vía específica participan en el Programa de Investigación:

b) funcionarios públicos de los agentes institucionales básicos, como son en nuestro país, CORPOTURISMO, CORZUTUR, OFICINA DE TURISMO DEL MUNICIPIO, y otros agentes claves en generación y fuentes de información estadísticas como son la OCEI (actual Instituto Nacional de Estadísticas) NACIONAL/ REGIONAL y el BANCO CENTRAL DE VENEZUELA NACIONAL/REGIONAL;

c) miembros activos y representantes de la ASOCIACIÓN DEEMPRESA TURÍSTICAS NACIONAL/REGIONAL, como otros agentes informantes y usuarios potenciales de la información turística resultante

d) miembro (s) representante(s) de la OMT, como agentes asesores técnicos de las CST

El número de participantes, los recursos físicos y económicos, se han de definir, según acuerdo entre las partes.

\section{Conclusiones y Recomendaciones}

Los aspectos considerados en el presente articulo constituyen en primer lugar una motivación para establecer un acercamiento de tipo institucional de LUZ a través del Programa de Investigación SIGTUR-ZULIA, con la CST de Venezuela que actualmente se encuentra en su primera fase inicio por parte ente Rector a nivel Nacional de la Actividad Turística (CORPOTURISMO), no sin antes concluir con la presentación a continuación de una serie de recomendaciones que permitirán establecer la viabilidad necesaria a la propuesta mencionada a fin de cooperar en el desarrollo de la CST de Venezuela.

a) establecer la Alianza Estratégica, bajo la figura de un CONVENIO INTERISTITUCIONAL MULTIPLE, con la participación de las ocho Instituciones propuestas:

b) propiciar la integración de los recursos humanos disponibles en las instituciones y ONG involucradas, para consolidar en un equipo técnico multidisciplinario y especializado en las áreas de la planificación del turismo, de la informática (SIG), economía, contaduría y estadísticas, entre otros;

c) organizar Talleres y Foros, para transferir a las instituciones involucradas, los alcances y beneficios de la elaboración de una CST para Venezuela, con la finalidad de sensibilizar al sector empresarial como agentes informantes básicos, y al resto de agentes participantes en el rol básico que les corresponde a cada uno de ellos ocupar;

exhortar a los agentes involucrados, a unir esfuerzos institucionales, para que a través del intercambio de los logros alcanzados o experiencias acumuladas, permitan que se amplíe el horizonte del conocimiento científico del turismo como actividad económica, capaz de coadyuvar con el desarrollo integral de los países, al ser producto de una planificación y gestión relacionada a otras actividades económicas y con enfoque integral y sustentable.

e) hacer del intercambio de las diversas organizaciones que participan en el desarrollo de las CST, en el ámbito internacional, un vinculo importante entre los países, dentro del cual se propague un lenguaje propicio para la solidaridad Institucional Internacional.

\section{Referencias}

CALDERA DE UGARTE NELLY. 2000. La conceptualización Teórica del SIGTUR-ZULIA-CONDES-LUZ 2000. Trabajo de Ascenso a la categoría de Profesor Titular, Universidad del Zulia, Maracaibo,Venezuela, 84 p. MEIS, SCOTT M. 2000. Documentos de las Cuentas Satélite de Turismo para la Conferencia CST200I. Disponíve

MILLER, RICHARD R. 2000. Simulated Satellite Acccounting Kesearch. $\wedge$ Decade of Commitment. WTTC/WEFA,

ORGANIZACIÓN MUNDIAL DEL TURISMO. 2000. LaCuenta Satelite de Turismo (CST). Un Proyecto Estratégico para la Organización Mundial del Turismo. Informe Secretario General OMT, Madrid, 15 p.

ORE Venezuela:ConsejoUniversitario de LUZ. V. XVII. 16 p.

Recibido en: 18/1/02

Aprobado en: 14/3/02 